\title{
The sialidase NEU3 promotes pulmonary fibrosis in mice
}

\author{
Darrell Pilling, Kyle Sahlberg, Tejas R. Karhadkar, Wensheng Chen, and Richard H. Gomer
}

\author{
Department of Biology \\ Texas A\&M University \\ 301 Old Main Drive
}

College Station, Texas. 77843-3474

Corresponding authors:

Darrell Pilling PhD or Richard H Gomer PhD

Phone: $979-458-5746$ or $979-458-5745$

E-mail: dpilling@bio.tamu.edu

E-mail:rgomer@tamu.edu 


\begin{abstract}
Sialic acid is often the distal sugar on glycoconjugates, and sialidases are enzymes that remove this sugar. In fibrotic lesions in human and mouse lungs, there is extensive desialylation of glycoconjugates, and upregulation of sialidases including the extracellular sialidase NEU3. In

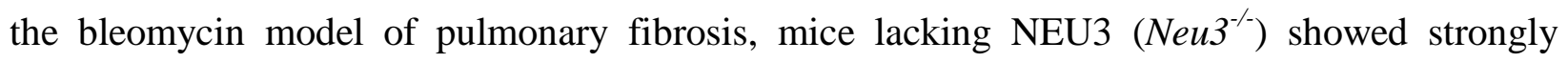
attenuated bleomycin-induced weight loss, lung damage, inflammation, upregulation of TGF- $\beta 1$, and fibrosis. This indicates that NEU3 is necessary for the full spectrum of bleomycin-induced pulmonary fibrosis. To determine if NEU3 is sufficient for fibrosis, mice not treated with bleomycin were treated with recombinant murine NEU3 or inactive NEU3. Aspiration of NEU3 caused inflammation and fibrosis in the lungs, while inactive NEU3 caused inflammation but not fibrosis. Mice were also treated with NEU3 starting 10 days after bleomycin. In male but not female mice, NEU3 increased inflammation and fibrosis. Inactive NEU3 did not enhance bleomycin-induced lung fibrosis. These results suggest that NEU3 is sufficient to induce fibrosis in the lungs, and that this effect is mediated by NEU3's enzymic activity.
\end{abstract}




\section{Introduction}

Fibrosing diseases, such as idiopathic pulmonary fibrosis (IPF), cardiac fibrosis, liver cirrhosis, and end-stage kidney disease, involve the progressive formation of scar tissue in internal organs that replaces the normal tissue, and are an increasing burden for healthcare systems [1-4]. IPF is a chronic and fatal disease that affects $\sim 3$ million people worldwide, with an incidence of 1 in $200>65$ years, and is characterized by fibrosis of the lungs and if untreated has a median survival of 3-5 years after initial diagnosis [5-7]. The only FDA-approved therapeutics, Nintedanib and Pirfenidone, slow but do not reverse the progression of the disease $[6,8]$.

Many secreted and cell-surface mammalian proteins are glycosylated, and many of the glycosylated structures have sialic acids as the monosaccharide at the end of the polysaccharide chain [9-11]. Sialidases, which are also called neuraminidases, remove the terminal sialic acid from these glycoconjugates [12, 13]. There are four known mammalian sialidases, NEU1, NEU2, NEU3, and NEU4, and each sialidase has different subcellular localizations and substrate specificities [12-14]. Of the four sialidases, only NEU3 is primarily extracellular [14-17]. This enzyme uses two tyrosines in the active site to remove sialic acid from glycoconjugates [13, 18].

The lungs from both preclinical models of lung fibrosis and patients with IPF have increased levels of sialidase activity in the bronchoalveolar lavage fluid (BALF), increased levels of NEU3 in the BAL, and increased levels of both NEU1 and NEU3 in the lung tissue [19-23]. Mice treated with NEU1 and NEU3 inhibitors, and $N e u 3^{--}$knockout mice, show strongly 
attenuated inflammation and fibrosis after bleomycin challenge [22-26], suggesting that NEU3 is necessary for the full extent of bleomycin-induced inflammation and fibrosis.

In this report, we assessed whether NEU3 might be sufficient to induce fibrosis. We previously found that mice have low levels of NEU3 $(\sim 1.7 \mathrm{ng})$ in their BALF, while mice treated with bleomycin have $\sim 15 \mathrm{ng}$ of NEU3 in their BALF at day 21 [23]. We find that aspiration of $15 \mathrm{ng}$ of mouse NEU3 every other day for 20 days caused inflammation and fibrosis in the lungs at day 21, and that the fibrosis was not observed using a mutated and thus inactive NEU3 lacking the two key tyrosines in the active site. We also find that NEU3 potentiates bleomycin-induced inflammation and pulmonary fibrosis. These results suggest that NEU3 is sufficient to induce fibrosis. 


\section{Materials and methods}

\section{Recombinant mouse NEU3 expression}

Chinese hamster ovary (CHO-K1) cells were cultured in CDM4CHO medium with Lglutamine (Cat\# SH30557.02, Hyclone/Cytiva, Marlborough, MA). Cells $\left(1 \times 10^{5}\right)$ were mixed with $2 \mu \mathrm{g}$ of $100 \mu \mathrm{g} / \mathrm{ml}$ of murine NEU3 expression plasmid (MR223297; Origene, Rockville, MD) in $100 \mu \mathrm{l}$ PBS (GE Lifesciences, Marlborough, MA) and were transfected by electroporation using a 4D-Nucleofector System (Lonza, Walkersville, MD) following the manufacturer's protocol. Before use, the plasmid was sequenced for verification as previously described [25, 27-29]. The transfected cells were kept at room temperature for 15 minutes for recovery, after which the CHO-K1 cells were cultured in $25 \mathrm{ml} \mathrm{CDM} 4 \mathrm{CHO}$ medium in a humidified incubator at $37^{\circ} \mathrm{C}$ with $5 \% \mathrm{CO}_{2}$. After 24 hours, $400 \mu \mathrm{g} / \mathrm{ml}$ of $\mathrm{G} 418(345812$; Calbiochem EMD, San Diego, CA) was added to select for transfected cells. After 10 days, the cells were collected and lysed, and c-Myc-tagged recombinant mouse NEU3 was purified using a Myc-Trap agarose kit (ytak-20; Chromotek, Hauppauge, NY) following the manufacturer's protocol. Recombinant protein was checked for protein concentration by OD 260/280/320 using a Take3 micro-volume plate with a SynergyMX plate reader (BioTek, Winooski, VT, USA). NEU3 is $51 \mathrm{kDa}$, and was further purified by centrifugation at $10,000 \mathrm{xg}$ for 5 minutes at $4^{\circ} \mathrm{C}$ through an Amicon Ultra $0.5 \mathrm{ml} 100 \mathrm{kDa}$ cutoff spin filter (Millipore, Billerica, MA, USA). The NEU3 was analyzed for size and purity by PAGE on, and Coomassie staining of, 4-20\% Tris/glycine gels (Bio-Rad, Hercules, CA, USA), as described previously [25, 27-29]. The NEU3 was stored in $50 \mu \mathrm{l}$ of $10 \%$ glycerol, $100 \mathrm{mM}$ glycine, and $25 \mathrm{mM}$ Tris- $\mathrm{HCl}, \mathrm{pH} 7.3$, at $4{ }^{\circ} \mathrm{C}$. 


\section{Recombinant mouse inactive NEU3 variant generation}

Starting with the murine NEU3 expression plasmid MR223297, a variant mutated to change the tyrosines at positions 179 and 181 to phenylalanines was generated using a QuikChange II Site-Directed Mutagenesis Kit (\#200523; Agilent, Santa Clara, CA) and the primer 5' CATCCCCGCCTTCGCCTTCTATGTCTCACGTTGG 3', with the underscored sequences representing the point mutation sites. Other workers found that these two mutations eliminate NEU3 sialidase activity [18]. The resulting plasmid was sequenced to confirm the point mutations and absence of other mutations.

\section{Cell Isolation and Culture}

Human peripheral blood was collected from healthy volunteers who gave written consent with specific approval from the Texas A\&M University human subjects review board. All methods were performed in accordance with the relevant guidelines and regulations. Blood collection, isolation of peripheral blood mononuclear cells (PBMC), and cell culture were done as described previously [22, 23, 25, 30]. Murine spleen cells were isolated by forcing diced spleen fragments through a $100 \mu \mathrm{m}$ cell strainer (BD Biosciences, San Jose, CA) using the plunger of a $1 \mathrm{ml}$ syringe (BD Medical, Franklin Lakes, NJ), as described previously [31]. To determine the activity of native and mutated NEU3 we assayed the ability of NEU3 to induce extracellular accumulation of IL-6 from human PBMC and murine spleen cells [23, 25], using human (BioLegend) and murine (PeproTech, Cranbury, NJ) IL-6 ELISA kits following the manufacturers' protocols. 


\section{Mouse models of pulmonary inflammation and fibrosis.}

This study was carried out in accordance with the recommendations in the Guide for the Care and Use of Laboratory Animals of the National Institutes of Health. The protocol was approved by Texas A\&M University Animal Use and Care Committee (IACUC 2020-0272). All procedures were performed under anesthesia, and all efforts were made to minimize suffering. Animals were housed with a 12-hour/12-hour light-dark cycle with free access to food and water, and all procedures were performed between 09:00 and noon. To induce inflammation and fibrosis, 7-8 week old 20-25 g male and female C57BL/6 mice (Jackson Laboratories, Bar Harbor, ME) were given an oropharyngeal aspiration of $3 \mathrm{U} / \mathrm{kg}$ bleomycin (2246-10; BioVision Incorporated, Milpitas, CA) in $50 \mu \mathrm{l}$ of $0.9 \%$ saline or oropharyngeal saline alone, as a control, as previously described [22, 23, 25, 32]. Starting 10 days after saline or bleomycin had been administered, some of the mice received an oropharyngeal aspiration of $15 \mathrm{ng}$ of recombinant mouse NEU3 or mutated NEU3 in $50 \mu 1$ of $0.9 \%$ saline, or saline, every 48 hours. An additional cohort of mice received only $15 \mathrm{ng}$ of recombinant mouse NEU3, mutated NEU3, or saline, every 48 hours over the course of 21 days. Three male and three female mice that did not receive saline, bleomycin, or NEU3 were defined as naïve mice. All the mice were monitored twice daily to observe any sign of distress. At the indicated time points, mice were euthanized by $\mathrm{CO}_{2}$ inhalation, and bronchoalveolar lavage fluid (BALF) and lung tissue was obtained and analyzed as described previously [22, 23, 25, 32, 33].

\section{Staining of bronchoalveolar lavage fluid (BALF) cells}


BALF cells were counted and processed to prepare cell spots as described previously [22, $23,25,32]$. After air drying for 48 hours at room temperature, some of the cell spots were fixed and immunochemistry was performed as described previously [22, 23, 25, 32] using anti-CD3 (NB600-1441, rabbit clone SP7, Novus Biologicals, Centennial, CO) to detect T-cells, antiCD11b (101202, clone M1/70, BioLegend, San Diego, CA) to detect blood and inflammatory macrophages, anti-CD11c (M100-3, clone 223H7, MBL International, Woburn, MA) to detect alveolar macrophages and dendritic cells, anti-CD45 (147702, clone I3/2.3, BioLegend) for total leukocytes, anti-Ly6g (127602, clone 1A8, BioLegend) to detect neutrophils, with isotypematched irrelevant rat (BioLegend) and rabbit (Novus Biologicals) antibodies as controls. Using a 40x objective, at least 150 cells from each stained BALF spot were examined and the percent positive cells was recorded.

\section{Lung histology}

After collecting BALF, the lungs from the mice were harvested and inflated with Surgipath frozen section compound (\#3801480, Leica, Buffalo Grove, IL), frozen on dry ice, and stored at $-80^{\circ} \mathrm{C} .10 \mu \mathrm{m}$ cryosections of lungs were placed on Superfrost Plus glass slides (VWR) and were air dried for 48 hours. Immunohistochemistry was done as previously described [22, 23, 25, 32] using anti-CD3, anti-CD11b, anti-CD11c, anti-CD45, anti-Ly6g, or anti-Mac2 (clone M3/38; BioLegend) antibodies with isotype-matched irrelevant rat and rabbit as controls. Positively stained cells were counted from randomly selected fields, and presented as the number of positive cells per $\mathrm{mm}^{2}$, as described previously [22, 23, 25, 32]. Lung sections were also stained with Sirius red to detect collagen and analyzed as previously described [23, 34]. 


\section{Statistical Analysis}

Statistical analysis was performed using Prism v7 (GraphPad Software, La Jolla, CA). Statistical significance between two groups was determined by t test, or between multiple groups using analysis of variance (ANOVA) with Sidak's post-test, and significance was defined as $\mathrm{p}<0.05$. 


\section{Results}

\section{Recombinant murine NEU3, but not mutated NEU3, has NEU3 activity}

We previously observed that the BALF from fibrotic lungs, and fibrotic lesions from human and mouse lungs, have elevated levels of NEU3, that NEU3 inhibitors attenuate bleomycin-induced pulmonary fibrosis, and that $N e u 3^{-/}$knockout mice are resistant to bleomycin-induced inflammation and fibrosis [22, 23, 25]. To further elucidate the contribution of NEU3 to fibrosis, we generated recombinant murine NEU3 and a mutated recombinant murine NEU3 protein with two point mutations in the active site. NEU3 and mutated NEU3 were $51 \mathrm{kDa}$ as assessed by PAGE and Coomassie staining (Figure 1A). Recombinant human NEU3 induces the accumulation of IL-6 from human peripheral blood mononuclear cells (PBMC), and this is blocked by NEU3 inhibitors [22, 23, 25], indicating that the effect on IL-6 is dependent on NEU3 activity. The recombinant murine NEU3, but not the mutated NEU3, upregulated extracellular accumulation of IL-6 in both human and murine cells (Figure 1B and C), indicating that the recombinant mouse NEU3 has NEU3 activity and that the mutated NEU3 does not and is thus inactive.

\section{Aspiration of NEU3 or inactive NEU3 induces inflammation}

To determine if elevated levels of NEU3 protein in the lung, without any other exogenous insult, could induce inflammation and/or fibrosis, mice received $15 \mathrm{ng}$ of either NEU3 or inactive NEU3 every 48 hours by oropharyngeal aspiration. This dose of NEU3 corresponds to the total amount of NEU3 in the BALF of mice at day 21 after bleomycin [23]. There was no discernable effect of NEU3 on the appearance or behavior of the mice, aspiration did not significantly affect body weight, or white fat, liver, kidney, spleen, heart, or brown fat weights as 
a percent of total body weight (Supplemental Figure 1A-C). Compared to naive controls, NEU3 aspiration increased BAL cell counts and BAL CD11c and CD45 cell counts in male and female mice (Figure 2). Compared to NEU3-treated mice, inactive NEU3-treated mice had lower BAL cell counts (Figure 2A), and this trend was observed in both male and female mice (Figure 2B). Compared to NEU3 aspiration, aspiration of inactive NEU3 caused a smaller increase in CD45 cells in the BAL for female but not male mice (Figure 2C and D). In female mice, NEU3 aspiration decreased the percentage of CD11c cells in the BAL compared to saline or inactive NEU3 aspiration (Figure 2F). There were no significant differences in BAL protein levels between naïve mice $(1.2 \pm 0.2 \mathrm{mg} / \mathrm{ml}$ : mean $\pm \mathrm{SEM})$, NEU3 $(1.8 \pm 0.4 \mathrm{mg} / \mathrm{ml}$; mean $\pm \mathrm{SEM})$ and inactive NEU3 $(1.4 \pm 0.9 \mathrm{mg} / \mathrm{ml}$; mean $\pm \mathrm{SEM})$ treated mice.

After removing BAL, lung sections were stained to detect immune cells that were not removed by lavage. In male mice, compared to naive mice, NEU3 increased the number of CD3, CD11b, CD45, and Mac2 positive cells in the lungs (Figure 3A-F). In female mice, NEU3 decreased CD3 positive cells and increased Mac2 and Ly6g positive cells (Figure 3E and F). Compared to NEU3 aspiration, aspiration of inactive NEU3 did not significantly affect counts of cells expressing any of the 6 markers. Together, the data indicate that aspiration of either form of NEU3 induces inflammation in the lungs of mice.

\section{Aspiration of NEU3 but not inactive NEU3 induces fibrosis}

Lung sections were also stained with picrosirius red to detect total collagen (Figure 4AF). In male and female mice, aspiration of NEU3 but not inactive NEU3 caused an increase in 
picrosirius red staining (Figure 4). These data suggest that NEU3 induces fibrosis, and that the effect is dependent on NEU3 activity.

\section{Following bleomycin, aspiration of NEU3 but not inactive NEU3 increases inflammation in male mice}

Bleomycin aspiration is a standard way to increase inflammation and fibrosis in the lungs of mice [35, 36], and we found that this also increases levels of NEU3 in the lungs $[22,23,25]$. To determine if further increasing levels of NEU3 in the lungs would alter inflammation and fibrosis after aspiration of bleomycin, mice were treated with saline or bleomycin at day 0 , and then from days 10 to 20 received 15 ng of either NEU3, inactive NEU3, or saline control every 2 days by oropharyngeal aspiration. As observed previously, male mice that received bleomycin have a drop in body weight between days 3 and 10, but there was no significant weight loss in the female mice (Figures 5A and B). Aspiration of NEU3 or inactive NEU3 did not significantly alter the weights of the mice (Figures 5A and B). As previously observed, compared to saline controls, bleomycin instillation led to an increase in the number of cells recovered from the BALF in both male and female mice at day 21 (Figure 5C and 5D). In the lungs of male mice that received bleomycin, NEU3 aspiration had significantly higher BAL cell counts compared to saline or inactive NEU3 treated mice (Figure 5C). In female mice that received either saline or bleomycin, NEU3 aspiration did not significantly alter BAL cell counts (Figure 5D).

In bleomycin-treated male but not female mice, aspiration of NEU3 but not inactive NEU3 increased the number of CD45 positive cells in the BAL at day 21 (Figures 6A and C). No treatment affected the percentages of CD3, CD11b, CD11c, CD45, or Ly6g positive cells in the 
BAL for male or female mice (Figures 6B and D). In the lungs of bleomycin-treated male mice after BAL, NEU3 aspiration, but not aspiration of inactive NEU3, increased the numbers of CD11c and CD45 positive cells (Figure 7A). Together, the results suggest that in male but not female mice, increasing levels of NEU3 starting at day 10 after bleomycin causes a further increase in inflammation.

\section{Aspiration of NEU3 but not inactive NEU3 for 10 days increases fibrosis}

Lung sections were also stained with picrosirius red to detect total collagen. In male and female mice, aspiration of NEU3, but not inactive NEU3, for 10 days increased picrosirius red staining (Figure 8M-N). In bleomycin treated mice, NEU3 aspiration increased picrosirius red staining in male but not female mice (Figure $8 \mathrm{M}-\mathrm{N}$ ). In male and female mice treated with bleomycin, compared to aspiration of NEU3, aspiration of inactive NEU3 reduced picrosirius red staining (Figure 8M-N). These data suggest that increasing NEU3 can increase fibrosis, and that this effect requires NEU3 activity. 


\section{Discussion}

In this report, we observed that the murine sialidase NEU3, but not enzyme inactive murine NEU3, can induce IL-6 production from human and murine cells as observed previously for human NEU3 [23, 37]. We also observed that NEU3 treatment increased the number of immune cells in the BAL and lung tissue both alone and after instillation of bleomycin, compared to naïve and saline treated mice respectively. Inactive NEU3 did increase the number of cells in the BAL compared to naïve mice, but the response was less than that of enzyme active NEU3. We also observed that in male mice, NEU3 augmented the number of cells in the BAL following bleomycin, and that inactive NEU3 did not augment the inflammatory response compared to active NEU3. NEU3 treatment also upregulated the fibrotic response in both male and female mice when given alone, but only NEU3, and not inactive NEU3, augmented the fibrotic response after bleomycin challenge.

Elevated NEU3 levels are found during inflammation and fibrosis in many organ

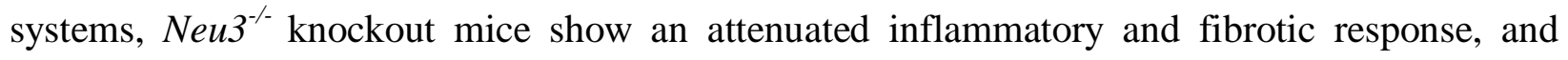
NEU3 inhibitors reduce these responses in wild type mice [19-26, 38-41]. We observed that the instillation of exogenous active NEU3, but not the inactive NEU3, augmented bleomycininduced lung inflammation in male mice, and fibrosis in male and female mice. This suggests that the observed effects of NEU3 on inflammation and fibrosis are due to the enzymatic activity of NEU3 rather than the presence of the NEU3 protein itself. Other workers, and this report, observed that compared to male mice, female mice have a reduced fibrotic response to bleomycin $([42,43]$ and Figure 8). We observed in this report that female mice have a reduced fibrotic response to aspirated NEU3 following bleomycin. This suggests that compared to male 
mice, female mice may have a reduced sensitivity to NEU3. Despite these sex differences, our results indicate that NEU3 potentiates pulmonary fibrosis. 


\section{Figure legends}

Figure 1: Recombinant murine NEU3, but not inactive NEU3, upregulates IL-6. A) Recombinant NEU3 proteins were purified (P) and then filtered through a $100 \mathrm{kDa}$ filter and the retentate $(>100)$ and flow through $(<100)$ were collected. Molecular mass markers in kDa are at left. B) Human PBMC and C) murine spleen cells were incubated with NEU3 or inactive NEU3 for two days. Supernatants were then assessed for B) human or C) murine IL-6 by ELISA. Values are mean \pm SEM, $n=3$.

\section{Supplementary Figure 1. Aspiration of NEU3 or inactive NEU3 had no significant effect on} body weight or organ weights. Percent change in body weight of A) male and B) female naïve mice, or mice after aspiration of NEU3 or inactive NEU3 for every 48 hours for 20 days. C) Weights of white fat, liver, kidneys, spleen, heart, and brown fat as percentage of total bodyweight at day 21 . Values are mean $\pm S E M, n=3$. There were no significant changes as determined by t test or one-way ANOVA, Sidak's test.

Figure 2: NEU3 treated mice have increased numbers of bronchoalveolar lavage (BAL) cells. A) The total number of cells in mouse BAL after the indicated treatment. Values are mean \pm SEM, n=6 (3 male and 3 female) mice per group. B) BAL cell counts separated into in male and female mice, $\mathrm{n}=3$ male and 3 female mice per group. $\mathbf{C}-\mathbf{F}$ ) BAL cell spots at day 21 were stained for the markers CD3, CD11b, CD11c, CD45, and Ly6g, and the percent of cells stained was determined in five randomly chosen fields of 100-150 cells, and the percentage was multiplied by the total number of BAL cells for that mouse to obtain the total number of BAL 
cells staining for the marker. Values are mean \pm SEM., $\mathrm{n}=3 .{ }^{*} \mathrm{p}<0.05 ; * * \mathrm{p}<0.01, * * * \mathrm{p}<$ 0.001 (one-way ANOVA, Sidak’s test).

Figure 3: Increase in immune cells in lungs post-BAL of NEU3 treated mice. Cryosections of mouse lungs were stained for A) CD3 B) CD11b, C) CD11c, D) CD45, E) Mac2 and F) Ly6g and counts were converted to the number of positive cells per $\mathrm{mm}^{2}$. Values are mean $\pm \mathrm{SEM}, \mathrm{n}=$ 3. ${ }^{*} \mathrm{p}<0.05 ; * * \mathrm{p}<0.01$ (one-way ANOVA, Sidak’s test).

Figure 4: NEU3 but not inactive NEU3 induces fibrosis. Sections of lung tissue from naïve mice and mice treated with NEU3 or inactive NEU3 were stained with picrosirius red to show collagen content. A) Naïve, B) NEU3, and C) inactive NEU3 treated male mice, and D) Naïve, E) NEU3, and F) inactive NEU3 treated female mice. All images are representative of three mice per group. Bar is $0.1 \mathrm{~mm}$. Picrosirius red quantification of $\mathbf{G}$ ) male and $\mathbf{H}$ ) female mice. Values are mean \pm SEM, $\mathrm{n}=3 .{ }^{*} \mathrm{p}<0.05 ; * * \mathrm{p}<0.01$ (one-way ANOVA, Sidak's test).

Figure 5: NEU3 increases the number of BAL cells in bleomycin treated male mice. Percent change in body weight in A) male and B) female mice after saline (S) or bleomycin (Bleo) aspiration at day 0 , and saline, NEU3, or inactive NEU3 treatment from days 10 to 20. Values are means \pm SEM, $n=3$ mice per group. The total number of cells in $\mathbf{C}$ ) male and $\mathbf{D})$ female mouse BAL after the indicated treatment. Values are mean $\pm \mathrm{SEM}, \mathrm{n}=3 .{ }^{*} \mathrm{p}<0.05 ; * * \mathrm{p}<0.01$ (one-way ANOVA, Sidak's test). 
Figure 6: NEU3, but not inactive NEU3, treated male mice have increased numbers of inflammatory immune cells in the BAL following bleomycin aspiration. Graphs show total number of BAL cells in A) male and B) female mice, and the percent of cells in C) male and D) female mice, staining for the markers CD3, CD11b, CD11c, CD45, and Ly6g. Values are mean \pm SEM, $\mathrm{n}=3 .{ }^{*} \mathrm{p}<0.05$ (one-way ANOVA, Sidak's test).

Figure 7: NEU3, but not inactive NEU3, treated male mice have increased numbers of inflammatory cells in lung tissue following bleomycin aspiration. Cryosections of A) male and B) female mouse lungs were stained for CD3, CD11b, CD11c, CD45, Mac2, and Ly6g, and counts were converted to the number of positive cells per $\mathrm{mm}^{2}$. Values are mean $\pm \mathrm{SEM}, \mathrm{n}=3$. $* p<0.05 ;$ (one-way ANOVA, Sidak’s test).

Figure 8: Enhanced fibrotic response in NEU3 treated mice following bleomycin. Sections of lung tissue from mice treated with saline or bleomycin (Bleo) aspiration at day 0, and NEU3 or inactive NEU3 treatment from days 10 to 20 , were stained with picrosirius red to show collagen content. A-F) Male and G-L) female mice aspirated with A and G) saline at day 0, B and $\mathbf{H}$ ) saline at day 0 and NEU3 from days 10-20, C and I) saline at day 0 and inactive NEU3 from days 10-20, D and J) bleomycin at day 0 and saline from days 10 to 20, $\mathbf{E}$ and $\mathbf{K}$ ) bleomycin at day 0 and NEU3 from days 10-20, F and L) bleomycin at day 0 and inactive NEU3 from days 10-20. All images are representative of three mice per group. Scale bar is 0.1 mm. Picrosirius red quantification of $\mathbf{M}$ ) male and $\mathbf{N}$ ) female mice. Values are mean \pm SEM, $\mathrm{n}=$ 3. $* \mathrm{p}<0.05 ; * * \mathrm{p}<0.01 * * * \mathrm{p}<0.001$ (one-way ANOVA, Sidak's test). 


\section{References}

1. Barnes PJ, Anderson GP, Fagerås M, Belvisi MG. Chronic lung diseases: prospects for regeneration and repair. European Respiratory Review. 2021;30(159):200213. doi: 10.1183/16000617.0213-2020.

2. Raimundo K, Chang E, Broder MS, Alexander K, Zazzali J, Swigris JJ. Clinical and economic burden of idiopathic pulmonary fibrosis: a retrospective cohort study. BMC Pulm Med. 2016;16:2. Epub 2016/01/07. doi: 10.1186/s12890-015-0165-1. PubMed PMID: 26732054; PubMed Central PMCID: PMCPMC4702364.

3. Sgalla G, Kulkarni T, Antin-Ozerkis D, Thannickal VJ, Richeldi L. Update in Pulmonary Fibrosis 2018. American Journal of Respiratory and Critical Care Medicine. 2019;200(3):292-300. doi: 10.1164/rccm.201903-0542UP.

4. Schattenberg JM, Lazarus JV, Newsome PN, Serfaty L, Aghemo A, Augustin S, et al. Disease burden and economic impact of diagnosed non-alcoholic steatohepatitis (nash) in five european countries in 2018: A cost-of-illness analysis. Liver Int. 2021. Epub 2021/02/17. doi: 10.1111/liv.14825. PubMed PMID: 33590598.

5. Raghu G, Rochwerg B, Zhang Y, Garcia CA, Azuma A, Behr J, et al. An Official ATS/ERS/JRS/ALAT Clinical Practice Guideline: Treatment of Idiopathic Pulmonary Fibrosis. An Update of the 2011 Clinical Practice Guideline. Am J Respir Crit Care Med. 2015;192(2):e3-19. Epub 2015/07/16. doi: 10.1164/rccm.201506-1063ST. PubMed PMID: 26177183.

6. Lederer DJ, Martinez FJ. Idiopathic Pulmonary Fibrosis. New England Journal of Medicine. 2018;378(19):1811-23. doi: 10.1056/NEJMra1705751. PubMed PMID: 29742380.

7. Cottin V, Spagnolo P, Bonniaud P, Nolin M, Dalon F, Kirchgässler K-U, et al. Mortality and Respiratory-Related Hospitalizations in Idiopathic Pulmonary Fibrosis Not Treated With Antifibrotics. Frontiers in Medicine. 2021;8(2815). doi: 10.3389/fmed.2021.802989.

8. Raghu G, Selman M. Nintedanib and Pirfenidone. New Antifibrotic Treatments Indicated for Idiopathic Pulmonary Fibrosis Offer Hopes and Raises Questions. American Journal of Respiratory and Critical Care Medicine. 2015;191(3):252-4. doi: 10.1164/rccm.201411-2044ED.

9. Schwab I, Nimmerjahn F. Intravenous immunoglobulin therapy: how does IgG modulate the immune system? Nat Rev Immunol. 2013;13(3):176-89. doi: 10.1038/nri3401. PubMed PMID: 23411799.

10. Freire-de-Lima L, Oliveira IA, Neves JL, Penha LL, Alisson-Silva F, Dias WB, et al. Sialic acid: a sweet swing between mammalian host and Trypanosoma cruzi. Front Immunol. 2012;3:356. doi: 10.3389/fimmu.2012.00356. PubMed PMID: 23230438; PubMed Central PMCID: PMC3515882.

11. Varki A, Gagneux P. Multifarious roles of sialic acids in immunity. Ann N Y Acad Sci. 2012;1253:16-36. doi: 10.1111/j.1749-6632.2012.06517.x. PubMed PMID: 22524423; PubMed Central PMCID: PMC3357316.

12. Monti E, Miyagi T. Structure and Function of Mammalian Sialidases. Top Curr Chem. 2015;366:183-208. Epub 2012/07/05. doi: 10.1007/128_2012_328. PubMed PMID: 22760823.

13. Smutova V, Albohy A, Pan X, Korchagina E, Miyagi T, Bovin N, et al. Structural Basis for Substrate Specificity of Mammalian Neuraminidases. PLoS ONE. 2014;9(9):e106320. doi: 10.1371/journal.pone.0106320.

14. Miyagi T, Yamaguchi K. Mammalian sialidases: Physiological and pathological roles in cellular functions. Glycobiology. 2012;22(7):880-96. doi: 10.1093/glycob/cws057.

15. Zanchetti G, Colombi P, Manzoni M, Anastasia L, Caimi L, Borsani G, et al. Sialidase NEU3 is a peripheral membrane protein localized on the cell surface and in endosomal structures. Biochemical Journal. 2007;408(2):211-9. doi: 10.1042/bj20070503.

16. Cirillo F, Ghiroldi A, Fania C, Piccoli M, Torretta E, Tettamanti G, et al. NEU3 Sialidase Protein Interactors in the Plasma Membrane and in the Endosomes. J Biol Chem. 2016;291(20):10615-24. Epub 
20160317. doi: 10.1074/jbc.M116.719518. PubMed PMID: 26987901; PubMed Central PMCID: PMCPMC4865910.

17. Rodriguez-Walker M, Daniotti JL. Human Sialidase Neu3 is S-Acylated and Behaves Like an Integral Membrane Protein. Sci Rep. 2017;7(1):4167. Epub 20170623. doi: 10.1038/s41598-017-04488w. PubMed PMID: 28646141; PubMed Central PMCID: PMCPMC5482835.

18. Albohy A, Li MD, Zheng RB, Zou C, Cairo CW. Insight into substrate recognition and catalysis by the human neuraminidase 3 (NEU3) through molecular modeling and site-directed mutagenesis. Glycobiology. 2010;20(9):1127-38. doi: 10.1093/glycob/cwq077.

19. Lambré CR, Pilatte $\mathrm{Y}$, Le Maho S, Greffard A, De Crémoux H, Bignon J. Sialidase activity and antibodies to sialidase-treated autologous erythrocytes in bronchoalveolar lavages from patients with idiopathic pulmonary fibrosis or sarcoidosis. Clin Exp Immunol. 1988;73(2):230-5. PubMed PMID: 3180512; PubMed Central PMCID: PMCPMC1541593.

20. Lillehoj EP, Hyun SW, Feng C, Zhang L, Liu A, Guang W, et al. Human airway epithelia express catalytically active NEU3 sialidase. Am J Physiol Lung Cell Mol Physiol. 2014;306(9):L876-86. Epub 2014/03/25. doi: 10.1152/ajplung.00322.2013. PubMed PMID: 24658138; PubMed Central PMCID: PMCPMC4010649.

21. Luzina IG, Lockatell V, Hyun SW, Kopach P, Kang PH, Noor Z, et al. Elevated expression of NEU1 sialidase in idiopathic pulmonary fibrosis provokes pulmonary collagen deposition, lymphocytosis, and fibrosis. Am J Physiol Lung Cell Mol Physiol. 2016;310(10):L940-54. Epub 2016/03/20. doi: 10.1152/ajplung.00346.2015. PubMed PMID: 26993524.

22. Karhadkar TR, Pilling D, Cox N, Gomer RH. Sialidase inhibitors attenuate pulmonary fibrosis in a mouse model. Scientific Reports. 2017;7(1):15069. Epub 2017/11/10. doi: 10.1038/s41598-017-15198-8. PubMed PMID: 29118338; PubMed Central PMCID: PMCPMC5678159.

23. Karhadkar TR, Chen W, Gomer RH. Attenuated pulmonary fibrosis in sialidase-3 knockout (Neu3(-/-)) mice. Am J Physiol Lung Cell Mol Physiol. 2020;318(1):L165-I79. Epub 2019/10/17. doi: 10.1152/ajplung.00275.2019. PubMed PMID: 31617733; PubMed Central PMCID: PMCPMC6985870.

24. Hyun SW, Liu A, Liu Z, Cross AS, Verceles AC, Magesh S, et al. The NEU1-selective sialidase inhibitor, C9-butyl-amide-DANA, blocks sialidase activity and NEU1-mediated bioactivities in human lung in vitro and murine lung in vivo. Glycobiology. 2016;26(8):834-49. Epub 2016/05/27. doi: 10.1093/glycob/cww060. PubMed PMID: 27226251.

25. Karhadkar TR, Meek TD, Gomer RH. Inhibiting sialidase-induced TGF- $\beta 1$ activation attenuates pulmonary fibrosis in mice. J Pharmacol Exp Ther. 2020. Epub 2020/11/05. doi: 10.1124/jpet.120.000258. PubMed PMID: 33144389.

26. Luzina IG, Lillehoj EP, Lockatell V, Hyun SW, Lugkey KN, Imamura A, et al. Therapeutic Effect of Neuraminidase-1-Selective Inhibition in Mouse Models of Bleomycin-Induced Pulmonary Inflammation and Fibrosis. Journal of Pharmacology and Experimental Therapeutics. 2021;376(1):136. doi: 10.1124/jpet.120.000223.

27. Crawford JR, Pilling D, Gomer RH. FcyRI mediates serum amyloid P inhibition of fibrocyte differentiation. Journal of Leukocyte Biology. 2012;92(4):699-711. Epub 2012/04/12. doi: 10.1189/jlb.0112033. PubMed PMID: 22493081; PubMed Central PMCID: PMC3441318.

28. Cox N, Pilling D, Gomer RH. Distinct Fcy Receptors Mediate the Effect of Serum Amyloid P on Neutrophil Adhesion and Fibrocyte Differentiation. The Journal of Immunology. 2014;193(4):1701-8. Epub 2014/07/16. doi: 10.4049/jimmunol.1400281. PubMed PMID: 25024390; PubMed Central PMCID: PMCPMC4120242.

29. Cox N, Pilling, D., Gomer, R. H. DC-SIGN activation mediates the differential effects of SAP and CRP on the innate immune system and inhibits fibrosis in mice Proceedings of the National Academy of Sciences. 2015;112(27):8385-90. Epub 2015/06/25. doi: 10.1073/pnas.1500956112. PubMed PMID: 26106150; PubMed Central PMCID: PMCPMC4500200. 
30. Pilling D, Fan T, Huang D, Kaul B, Gomer RH. Identification of markers that distinguish monocytederived fibrocytes from monocytes, macrophages, and fibroblasts. PLoS ONE. 2009;4(10):e7475. Epub 2009/10/17. doi: 10.1371/journal.pone.0007475. PubMed PMID: 19834619; PubMed Central PMCID: PMC2759556.

31. Crawford JR, Pilling D, Gomer RH. Improved serum-free culture conditions for spleen-derived murine fibrocytes. Journal Of Immunological Methods. 2010;363(1):9-20. Epub 2010/10/05. doi: DOI: 10.1016/j.jim.2010.09.025. PubMed PMID: 20888336; PubMed Central PMCID: PMC2997166

32. Pilling D, Gomer RH. Persistent Lung Inflammation and Fibrosis in Serum Amyloid P Component (Apcs -/-) Knockout Mice. PLoS ONE. 2014;9(4):e93730. Epub 2014/04/04. doi: 10.1371/journal.pone.0093730. PubMed PMID: 24695531; PubMed Central PMCID: PMCPMC3973556.

33. Daubeuf F, Frossard N. Performing Bronchoalveolar Lavage in the Mouse. Current Protocols in Mouse Biology: John Wiley \& Sons, Inc.; 2012. p. 167-75.

34. Pilling D, Roife D, Wang M, Ronkainen SD, Crawford JR, Travis EL, et al. Reduction of bleomycininduced pulmonary fibrosis by serum amyloid P. The Journal of Immunology. 2007;179(6):4035-44. Epub 2007/09/06. doi: 10.4049/jimmunol.179.6.4035. PubMed PMID: 17785842; PubMed Central PMCID: PMCPMC4482349.

35. Tashiro J, Rubio GA, Limper AH, Williams K, Elliot SJ, Ninou I, et al. Exploring Animal Models That Resemble Idiopathic Pulmonary Fibrosis. Frontiers in Medicine. 2017;4(118). doi: 10.3389/fmed.2017.00118.

36. Moore BB, Hogaboam CM. Murine models of pulmonary fibrosis. Am J Physiol Lung Cell Mol Physiol. 2008;294(2):L152-60. Epub 2007/11/13. doi: 00313.2007 [pii]

10.1152/ajplung.00313.2007. PubMed PMID: 17993587.

37. Sundararaj K, Rodgers JI, Marimuthu S, Siskind L, Bruner E, Nowling TK. Neuraminidase activity mediates IL- 6 production by activated lupus-prone mesangial cells. American Journal of PhysiologyRenal Physiology. 2017;314(4):F630-F42. doi: 10.1152/ajprenal.00421.2017.

38. Pilling D, Karhadkar TR, Gomer RH. High-fat diet-induced adipose tissue and liver inflammation and steatosis in mice are reduced by inhibiting sialidases. The American Journal of Pathology. 2020;191(1):131-43. Epub 2020/10/12. doi: 10.1016/j.ajpath.2020.09.011. PubMed PMID: 33039353; PubMed Central PMCID: PMCPMC7786079.

39. Pilling D, Karhadkar TR, Gomer RH. A CD209 ligand and a sialidase inhibitor differentially modulate adipose tissue and liver macrophage populations and steatosis in mice on the Methionine and Choline-Deficient (MCD) diet. PLOS ONE. 2020;15(12):e0244762. Epub 2020/12/31. doi: 10.1371/journal.pone.0244762. PubMed PMID: 33378413; PubMed Central PMCID: PMCPMC7773271.

40. Yang WH, Westman JS, Heithoff DM, Sperandio M, Cho JW, Mahan MJ, et al. Neu3 neuraminidase induction triggers intestinal inflammation and colitis in a model of recurrent human food-poisoning. Proc Natl Acad Sci U S A. 2021;118(29). doi: 10.1073/pnas.2100937118. PubMed PMID: 34266954; PubMed Central PMCID: PMCPMC8307618.

41. Yang WH, Heithoff DM, Aziz PV, Haslund-Gourley B, Westman JS, Narisawa S, et al. Accelerated Aging and Clearance of Host Anti-inflammatory Enzymes by Discrete Pathogens Fuels Sepsis. Cell Host Microbe. 2018;24(4):500-13.e5. doi: 10.1016/j.chom.2018.09.011. PubMed PMID: 30308156; PubMed Central PMCID: PMCPMC6223661.

42. Redente EF, Jacobsen KM, Solomon JJ, Lara AR, Faubel S, Keith RC, et al. Age and sex dimorphisms contribute to the severity of bleomycin-induced lung injury and fibrosis. Am J Physiol Lung Cell Mol Physiol. 2011;301(4):L510-8. Epub 20110708. doi: 10.1152/ajplung.00122.2011. PubMed PMID: 21743030; PubMed Central PMCID: PMCPMC3191751.

43. Voltz JW, Card JW, Carey MA, Degraff LM, Ferguson CD, Flake GP, et al. Male sex hormones exacerbate lung function impairment after bleomycin-induced pulmonary fibrosis. Am J Respir Cell Mol 
bioRxiv preprint doi: https://doi.org/10.1101/2022.02.12.480197; this version posted February 12,2022 . The copyright holder for this preprint (which was not certified by peer review) is the author/funder. All rights reserved. No reuse allowed without permission.

Biol. 2008;39(1):45-52. Epub 20080214. doi: 10.1165/rcmb.2007-03400C. PubMed PMID: 18276795; PubMed Central PMCID: PMCPMC2438447. 
Figure 1

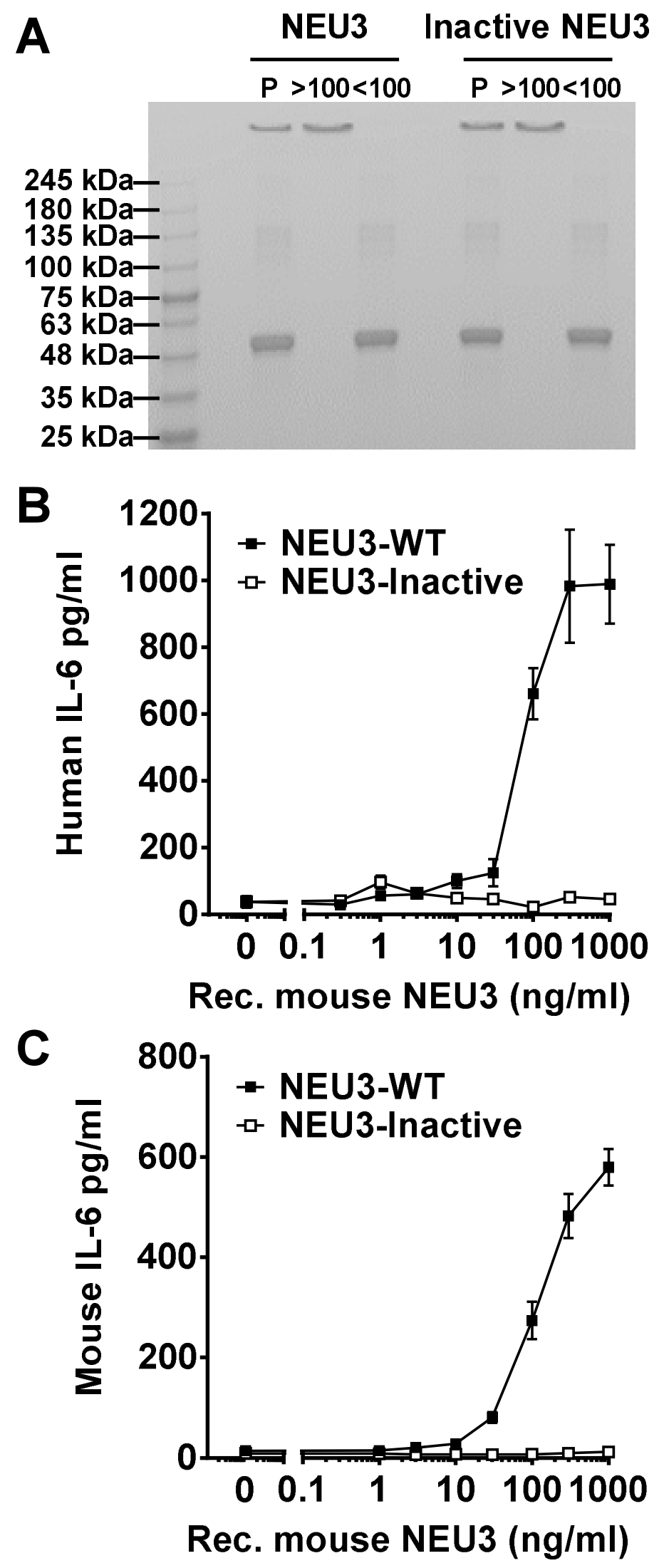



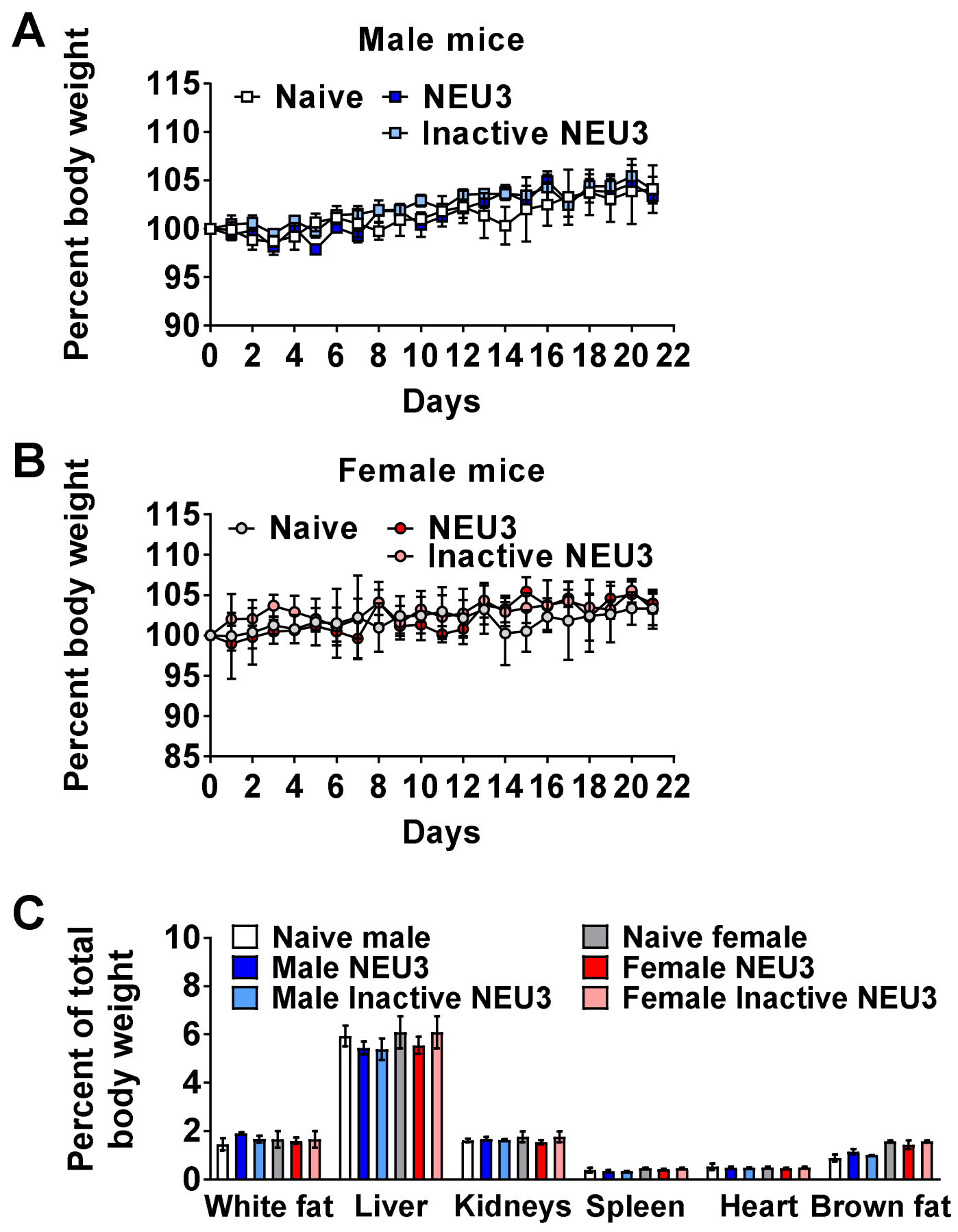
Figure 2

A

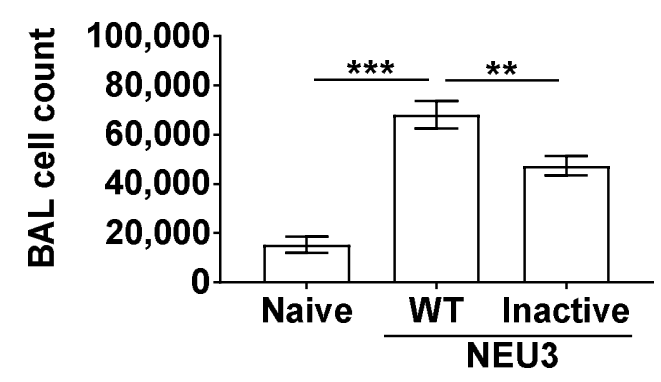

C

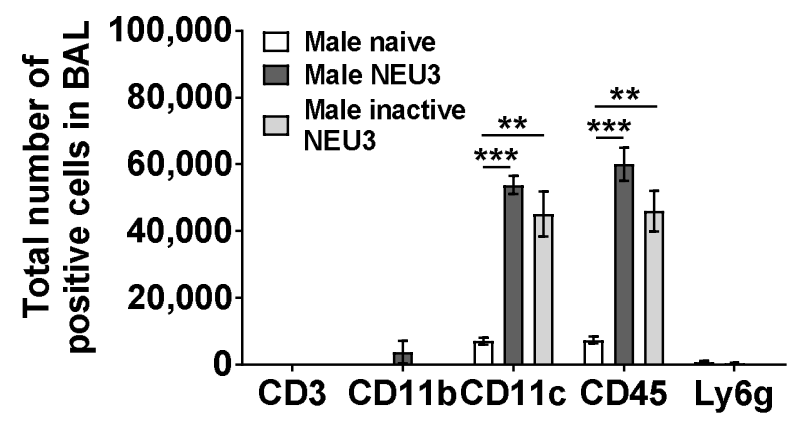

E

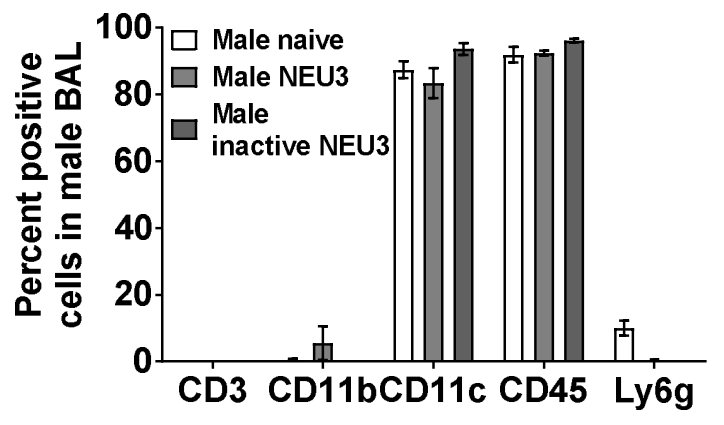

B
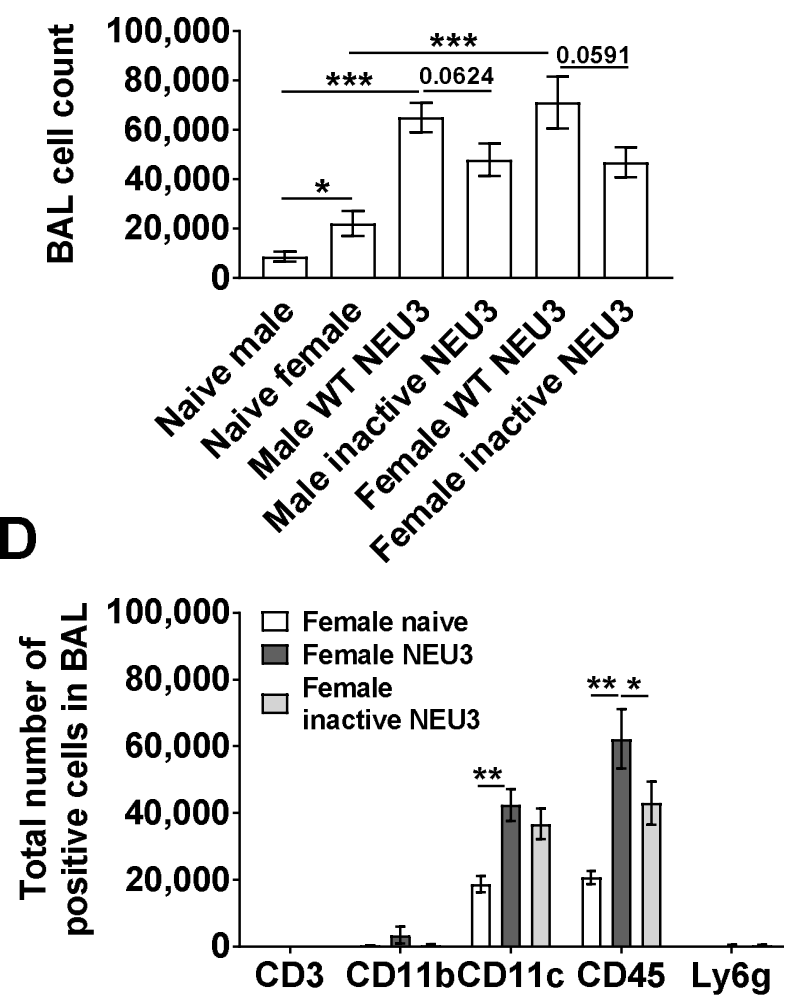

F

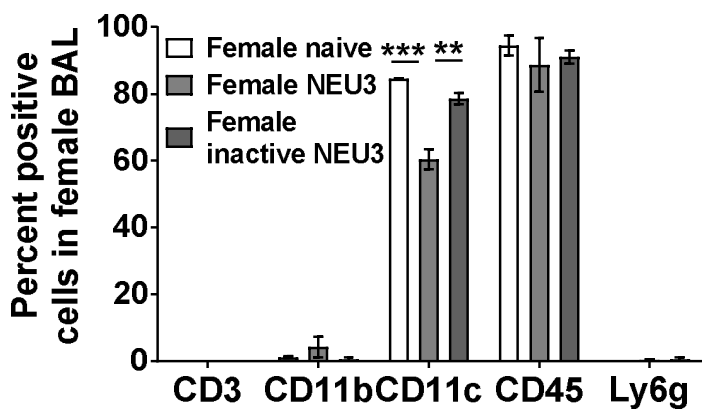


Figure 3

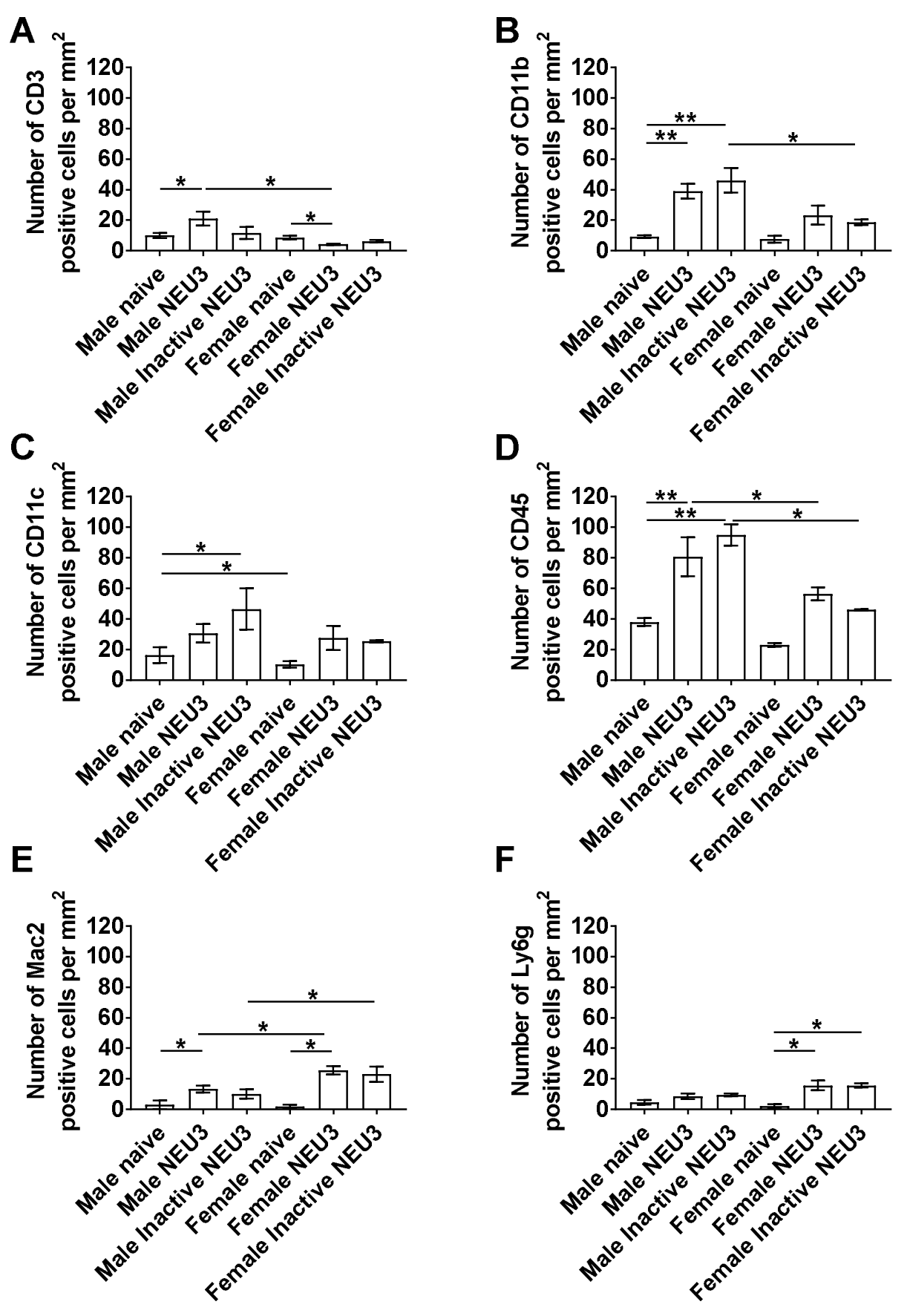


Figure 4
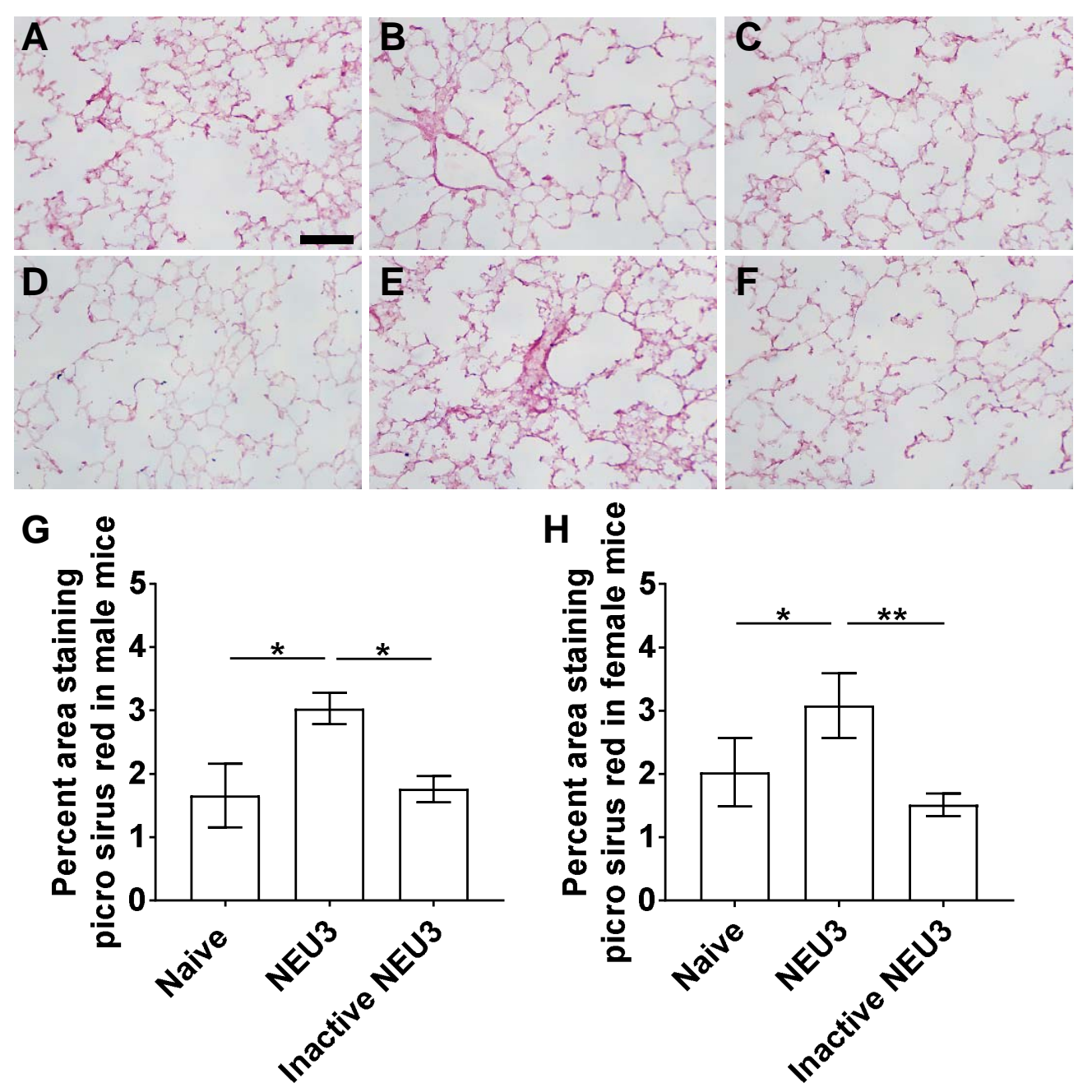
Figure 5

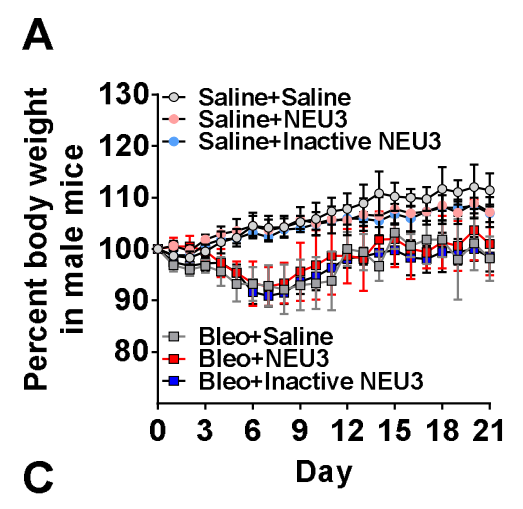

B
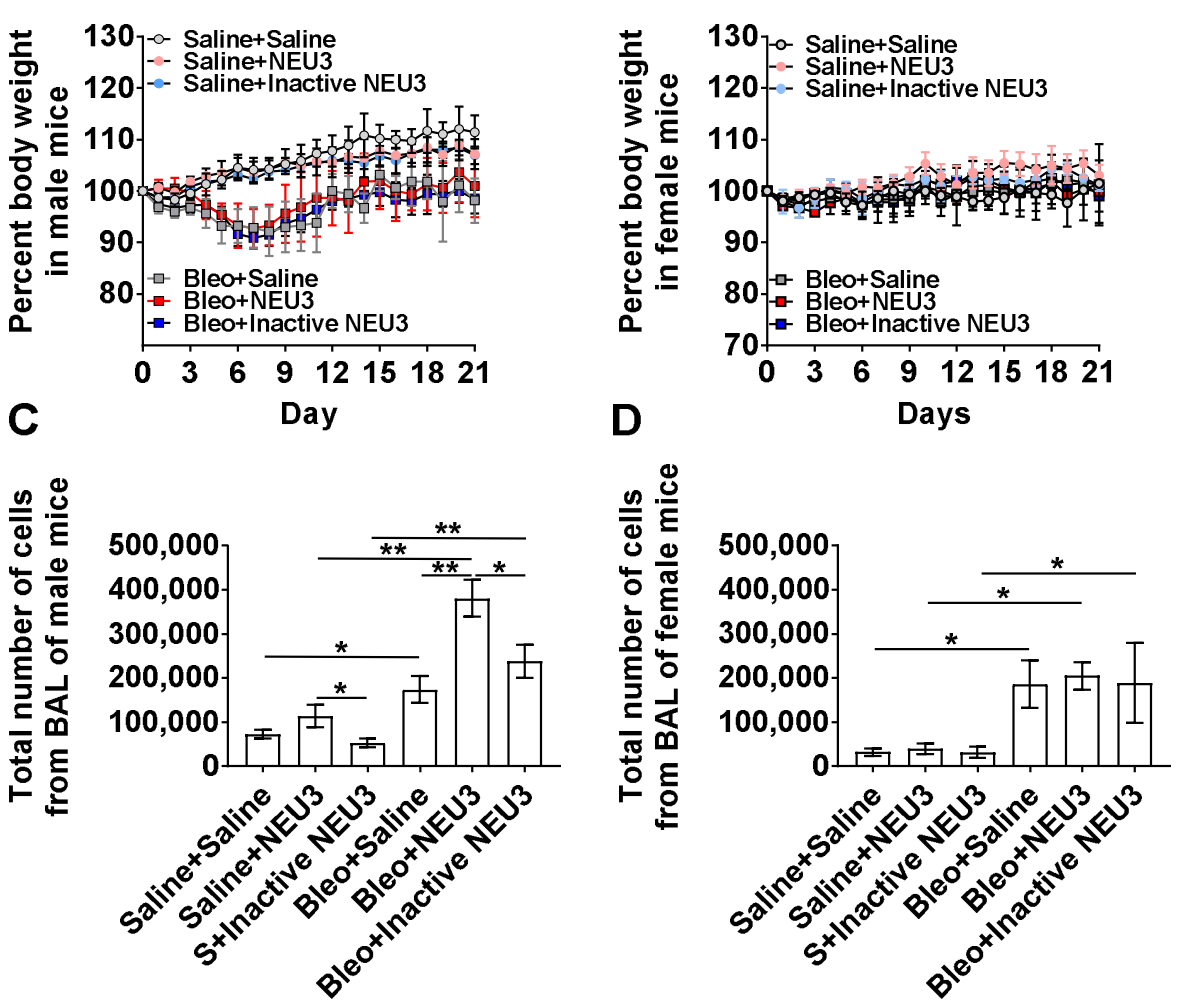
Figure 6
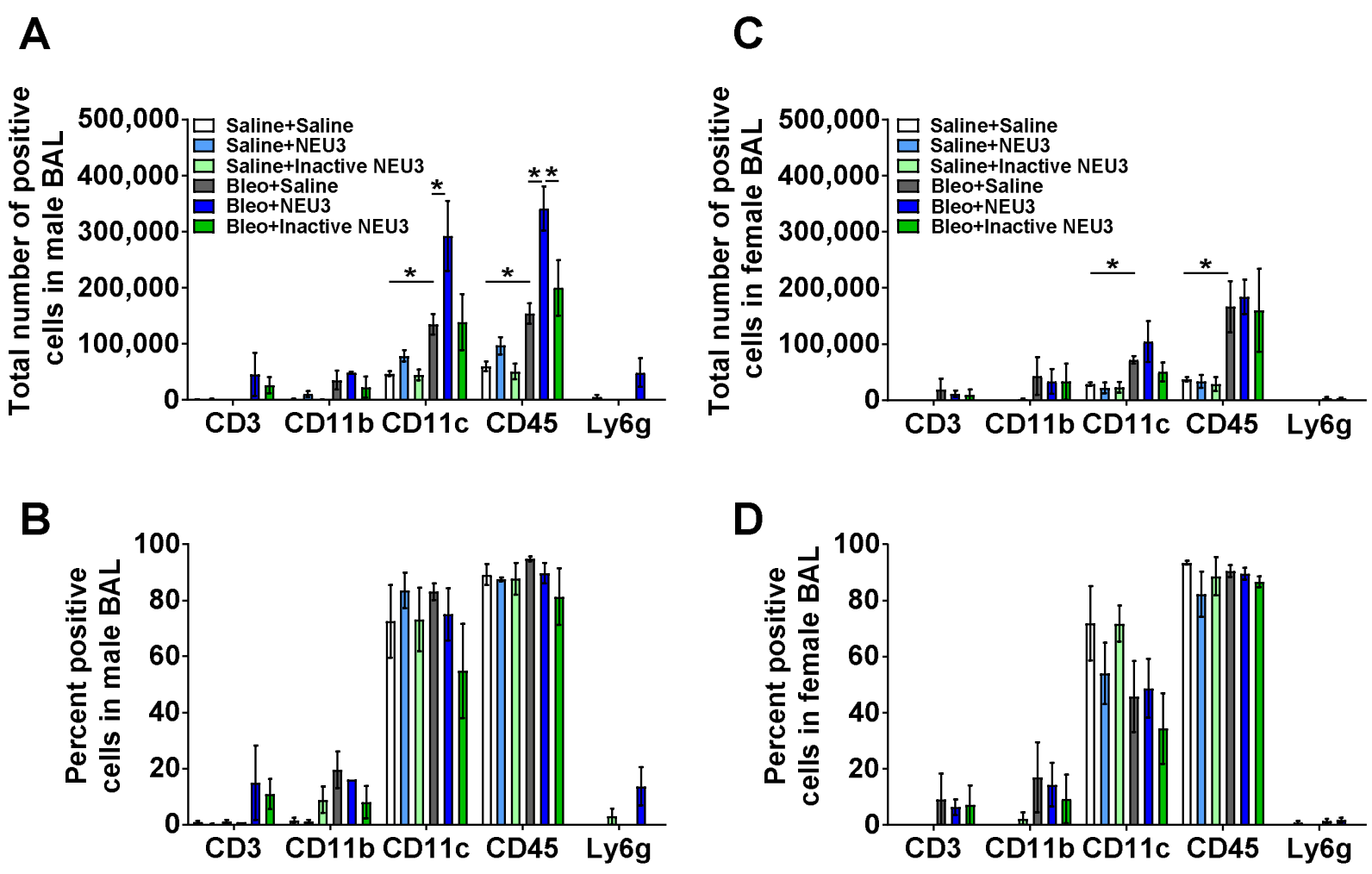
Figure 7 IHC analysis

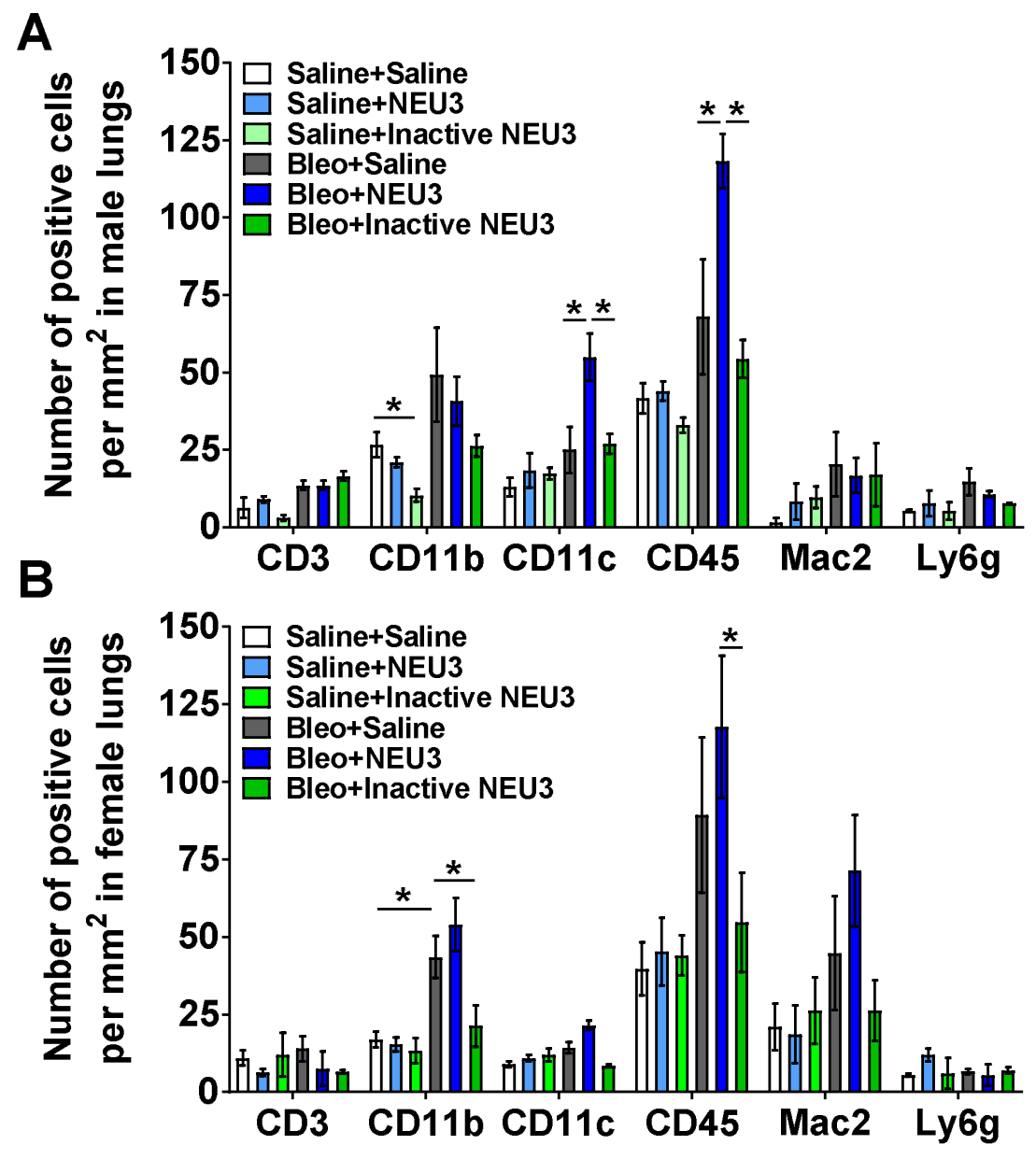


Figure 8
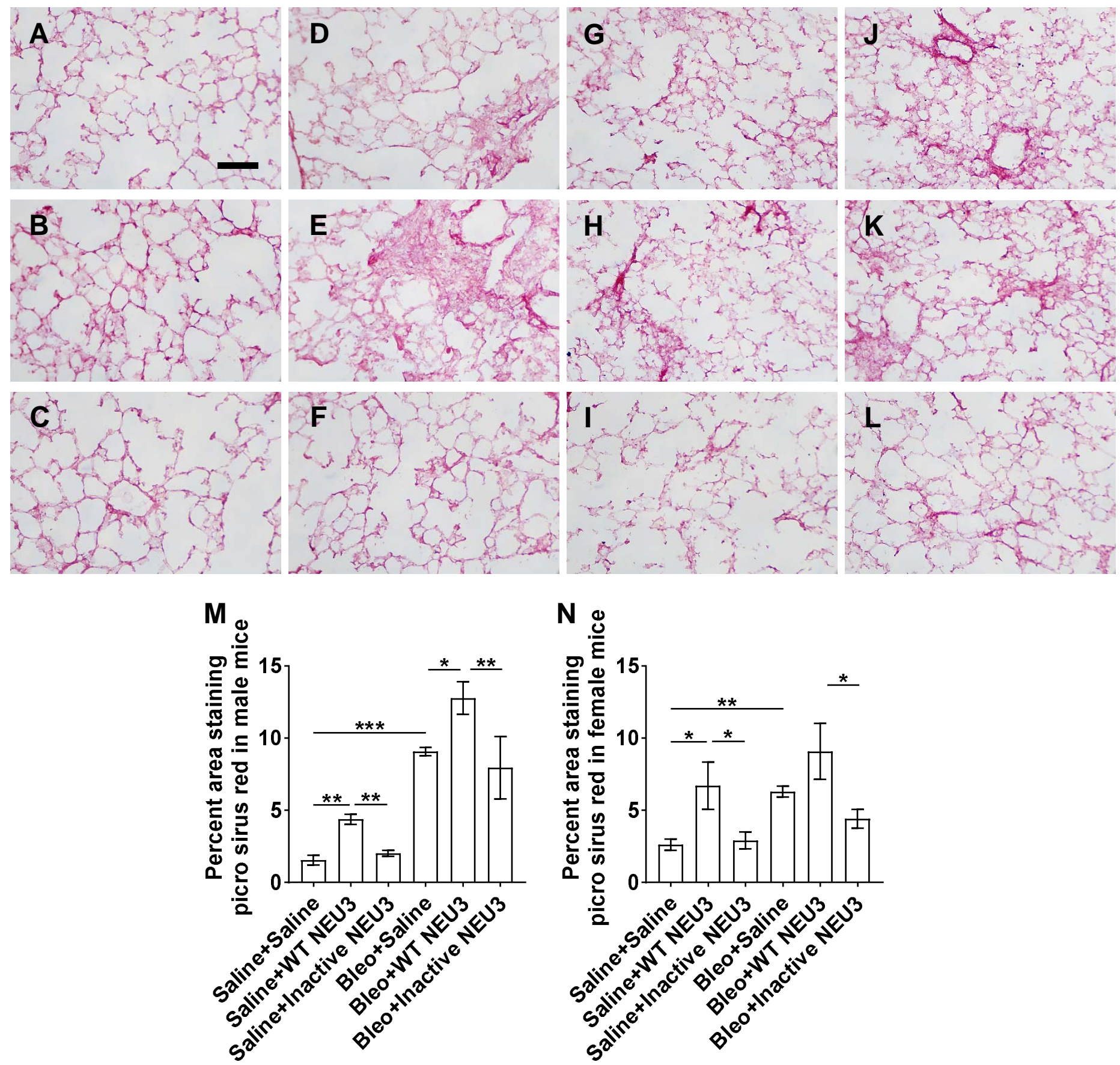